Article de recherche

\title{
Responsabilité sociétale de l'entreprise : une étude de la nature et de la valeur perçues des ressources mobilisées dans trois entreprises du secteur de l'imprimerie.
}

\author{
Marie DA FONSECA ${ }^{9}$ et Elise BONNEVEUX ${ }^{10}$
}

\section{Résumé :}

Cette recherche exploratoire porte sur les ressources mobilisées par les entreprises pour mettre en auvre une démarche responsable. Notre objectif est de sortir de l'analyse des success story d'entreprises pour proposer une grille d'intelligibilité des divergences d'approches en vigueur dans les entreprises d'un même secteur. Plus particulièrement, nous nous intéressons à la nature et à la valeur des ressources sollicitées tout en en questionnant les différences de perception en fonction de la stratégie de l'entreprise. Nous présentons les résultats d'une étude de cas menée dans trois imprimeries familiales. La collecte des données s'est appuyée sur une série de trois entretiens semi-directifs par entreprise avec les dirigeants et le recueil de données secondaires. Nous avons ensuite procédé à une analyse de contenu thématique. Nos résultats font principalement ressortir qu'une même ressource peut être perçue différemment en fonction du contexte et des acteurs qui la mobilisent. Ils débouchent sur la proposition d'une modélisation du processus de construction de la valeur d'une ressource sollicitée dans le cadre d'une démarche de responsabilité sociétale de l'entreprise (RSE).

Mots clés : Responsabilité sociétale des entreprises, théorie fondée sur les ressources, dirigeant d'entreprise familiale.

\footnotetext{
${ }^{9}$ Maître de conférences, Sciences de Gestion, Université de Savoie, IREGE (EA 2426) marie.da-fonseca@univ-smb.fr

${ }^{10}$ Maître de conférences, Sciences de Gestion, Université de Tours, VALLOREM (EA 6296) elise.bonneveux@univ-tours.fr
}

RIMHE - Revue Interdisciplinaire Management, Homme \& Entreprise n³2 - Eté 2018 
Article de recherche : Responsabilité sociétale de l'entreprise : une étude de la nature et de la valeur perçues des ressources mobilisées dans trois entreprises du secteur de

l'imprimerie - Marie DA FONSECA et Elise BONNEVEUX

\begin{abstract}
This exploratory research focuses on the resources mobilized by companies to implement a responsible approach. Our objective is to path out of success stories and propose a grid of intelligibility of divergent approaches used by companies of the same sector. We are particularly interested in the nature and value of the solicited resources while questioning the differences in perception according to the strategy of the company. We present the results of a case study conducted in three family businesses within the printing sector. Data collection is based on a series of three semi-structured interviews per company and secondary data collection. We then proceeded to a thematic content analysis. Our results highlight that the same resource can be perceived differently depending on the context and the actors who mobilize it. They lead to a model proposition of the resource value-building process in a corporate social responsibility (CSR) approach.
\end{abstract}

\title{
Keywords:
}

Corporate social responsibility, resource-based theory, manager of family company. 
Article de recherche : Responsabilité sociétale de l'entreprise : une étude de la nature et de

la valeur perçues des ressources mobilisées dans trois entreprises du secteur de

l'imprimerie - Marie DA FONSECA et Elise BONNEVEUX

\section{Introduction}

L'étude présentée dans cet article porte sur la nature et la valeur des ressources mobilisées dans le cadre d'une démarche responsable. Selon Acquier (2010), la Responsabilité Sociétale de l'Entreprise (RSE) constitue un véritable défi managérial et organisationnel. Il considère qu'afin d'enrichir ce champ de la littérature, les implications managériales liées à la responsabilité sociétale doivent être analysées et interprétées dans une perspective dynamique (Acquier, 2007 ; Acquier et Dalmasso, 2009). La RSE peut ainsi être abordée comme un changement organisationnel nécessitant la mobilisation, le développement ou encore l'acquisition de nouvelles ressources. L'objectif est de mieux comprendre le fonctionnement et le comportement des organisations. Pour cela, après une revue de la littérature sur la RSE et les théories basées sur les ressources, nous avons suivi les recommandations de Weppe, Warnier et Lecocq (2013) de ne pas se focaliser exclusivement sur les ressources stratégiques qui confèrent un avantage concurrentiel, mais de s'intéresser également aux ressources ordinaires ou négatives. Selon ces auteurs, la valeur de la ressource serait liée à la perception du dirigeant, et son impact sur la performance varierait en fonction de l'utilisation qui en sont faite. Ainsi, une ressource stratégique serait perçue par les entreprises comme positive car elle est une source potentielle de rente. Les ressources ordinaires sont les ressources perçues comme les actifs communs à plusieurs entreprises d'un même marché et présentent un effet neutre sur la performance. Les ressources négatives, quant à elles, sont perçues comme des ressources peuvant freiner ou détruire la capacité de la firme à générer des rentes. Elles sont généralement peu prisées par les entreprises du secteur. Nos questions de recherche sont ainsi : quelles sont les ressources mobilisées dans le cadre d'une démarche de RSE ? Une même ressource peut-elle être perçue comme stratégique, ordinaire ou négative par les dirigeants d'entreprises comparables ? Comment se construit la valeur d'une ressource au sein d'une entreprise dans le cadre d'une démarche responsable ?

Pour apporter des éléments de réponse à cette problématique, nous avons procédé à une étude de cas de trois entreprises familiales du secteur de l'imprimerie. Nos résultats font ressortir la variabilité de la perception de la nature et de la valeur d'une ressource en fonction du contexte et des dirigeants de l'entreprise. Leur principale contribution est d'éclairer le processus de construction de la valeur d'une ressource dans le cadre la mise en œuvre d'une démarche RSE.

\section{Enjeux de la recherche et cadre théorique}

Dans cette partie, nous présentons une revue de littérature sur la responsabilité sociétale des entreprises et l'approche théorique fondée sur les ressources pour, dans un troisième temps approfondir le lien entre les deux. Nous explicitons le 
Article de recherche : Responsabilité sociétale de l'entreprise : une étude de la nature et de

la valeur perçues des ressources mobilisées dans trois entreprises du secteur de

l'imprimerie - Marie DA FONSECA et Elise BONNEVEUX

positionnement de l'étude dans l'approche de Weppe, Warnier et Lecocq (2013), tout en clarifiant ses objectifs.

\subsection{Les enjeux de la mise en œuvre d'une démarche de responsabilité sociétale}

La responsabilité sociétale est un concept dont la naissance académique est attribuée à Bowen (1953), et dont les contours et les définitions n'ont eu de cesse d'évoluer (Frederick, 1978 ; Gendron, 2000). Plusieurs approches théoriques sont identifiées : éthique, managériale et critique (Gond et Matten, 2007). Pour autant, malgré la multiplication des travaux, le concept de responsabilité sociétale n'est pas encore stabilisé. Pour Wood (1991), il faut sortir du seul aspect philosophique de la RSE et la rendre pragmatique et opérationnelle. L'auteur propose d'analyser les comportements que les entreprises adoptent en réponse aux attentes sociétales. Les comportements organisationnels sont toutefois dépendants des capacités de l'entreprise à réagir, mais aussi de sa volonté à changer. Cela implique que les entreprises doivent, en prenant en compte les enjeux écologiques et sociaux, (re)penser la production de leurs biens et/ou services et les conditions de cette production (Acquier et Gond, 2005). Ainsi, la mise en œuvre d'une démarche de responsabilité sociétale ne se limite pas à l'introduction de nouveaux outils mais nécessite d'envisager une véritable réflexion stratégique.

La notion de responsabilité sociétale implique que les entreprises se préoccupent d'enjeux qui vont au-delà de leur sphère d'activité directe. De ce fait, développer une démarche de responsabilité sociétale entraîne une réflexion et des changements dans la structuration de l'organisation. Certaines entreprises choisissent à ce titre de créer une fonction ou un service développement durable (Capron et Quairel-Lanoizelée, 2004) dédié à l'opérationnalisation de ces changements. Toutefois, de par l'absence de définition concrète de ce qu'est la responsabilité sociétale et d'un cadre structurant, les actions menées dans les entreprises sont bien souvent l'œuvre d'un mode de pilotage intuitif, focalisé sur la vision de l'acteur qui a en charge cette mission. Nielsen et Thomsen (2007) montrent que dans le contexte des petites et moyennes entreprises (PME), l'appropriation de la responsabilité sociétale est ancrée dans les valeurs personnelles du dirigeant. Toutefois, selon Paradas (2007), cet engagement, fondé sur les valeurs, ne permet pas de lever tous les freins managériaux (Acquier, 2010; Havard et Ingham, 2017). Ceux-ci peuvent être internes ou externes et être liés à un manque de ressources à la fois humaines, financières (Spence et al., 1998) et/ou à un manque de temps, de connaissances ou d'informations (Clark, 2000).

Afin d'étudier comment les dirigeants de PME en charge de la responsabilité sociétale parviennent à lever ces freins et la mettre en œuvre, il convient de s'intéresser aux pratiques des entreprises. Leur vulnérabilité économique, le manque de ressources financières ainsi qu'un déficit en compétences et en temps renforcent la tendance des dirigeants à se focaliser sur une perspective à court-terme (Auberger et Quairel, 2004),

RIMHE - Revue Interdisciplinaire Management, Homme \& Entreprise

n³2 - Eté 2018

- 48 - 
Article de recherche : Responsabilité sociétale de l'entreprise : une étude de la nature et de

la valeur perçues des ressources mobilisées dans trois entreprises du secteur de

l'imprimerie - Marie DA FONSECA et Elise BONNEVEUX

alors que les démarches responsables nécessitent une projection dans le temps et une vision sur le long terme.

Ainsi, l'engagement responsable donne naissance à des situations contrastées qui peuvent être liées aux représentations que se font les acteurs et aux possibilités opérationnelles dont ils disposent (Maon et al., 2008 ; Hine et Preuss, 2009 ; Pedersen, 2010). La structure organisationnelle, la structure du capital et de la gouvernance (Johnson et Greening, 1999), la mobilisation des compétences (Mathieu et Soparnot, 2009), la culture organisationnelle (Sharma, 2000) ou encore les caractéristiques de l'activité (Marais et Reynaud, 2008) sont autant de facteurs qui interfèrent dans la prise de décision des dirigeants. Par ailleurs, il ne faut pas négliger l'influence de l'écosystème dans lequel évolue l'entreprise, et les pressions, plus ou moins coercitives, que certaines de ses composantes peuvent exercer (King et Lenox, 2000 ; Milsein et al., 2002).

La responsabilité sociétale représente également une opportunité pour l'entreprise, un levier de développement, ce qui en fait un objectif de gestion. Dans une logique de RSE, les trois piliers -économique, environnemental, social- ne sont pas hiérarchisés (Saulquin et Schier, 2007). Cela implique d'avoir une stratégie globale en mettant sur le même plan l'ensemble des dimensions de la RSE. La démarche ainsi développée par l'entreprise permet de soutenir les dynamiques interne et externe. Comment les dirigeants incluent-ils les ressources de l'entreprise dans la définition et la déclinaison des objectifs multiples associés à la mise en œuvre d'une démarche responsable ?

\subsection{L'approche par les ressources comme grille d'intelligibilité}

Pour répondre à cette question de recherche, nous avons sollicité l'approche théorique fondée sur les ressources (Resource Based View), qui domine en management stratégique (Barney, 2001 ; Métais, 2004 ; Weppe et al., 2013). Cette approche considère que les entreprises sont dotées d'actifs tangibles, intangibles et renouvelables (Wernerfelt, 1984 ; Barney, 1991 ; Peteraf et Barney, 2003), et possèdent un avantage concurrentiel, dès lors qu'elles parviennent à acquérir des ressources de valeur, c'est-à-dire valorisables, rares, inimitables et non-substituables (VRIN). Cette approche a donné lieu à de nombreux débats scientifiques notamment avec Barney (2001). Porter (1991) ou encore Priem et Butler (2001) ont ainsi pointé la difficulté de mise en œuvre de la théorie, de par le caractère tautologique de la question de la valeur des ressources et de son lien avec la performance. L'approche a ensuite été enrichie par l'introduction des notions de compétences, de connaissances ou encore de capacités dynamiques. La difficile catégorisation des ressources n'en constitue pas moins une de ses principales critiques (Acquier et Dalmasso, 2009). La notion de ressource est présentée comme un stock d'actifs disponibles, possédés ou contrôlés par une firme (Amit et Schoemaker, 1993). Cette définition a donné lieu à

RIMHE - Revue Interdisciplinaire Management, Homme \& Entreprise

n³2 - Eté 2018 
Article de recherche : Responsabilité sociétale de l'entreprise : une étude de la nature et de

la valeur perçues des ressources mobilisées dans trois entreprises du secteur de

l'imprimerie - Marie DA FONSECA et Elise BONNEVEUX

des travaux qui participent à la catégorisation des ressources, en fonction notamment du contexte industriel étudié (Miller et Shamsie, 1996). Helfat et Peteraf (2003, p. 999) soulignent que le concept de ressource «fait référence à un actif ou matière première (tangible ou intangible) qu'une organisation possède, contrôle, ou auquel elle a accès de façon quasi permanente » afin de concevoir et mettre en œuvre sa stratégie. Afin d'obtenir un avantage concurrentiel, l'entreprise doit acquérir des ressources durables et spécifiques, autrement dit non transférables et immobiles (Conner et Prahalad, 1996). Les entreprises sont considérées comme des lieux au sein desquels se combinent des ressources physiques (usines, équipements, matières premières, etc), humaines (salariés), mais également des ressources intangibles tels que les savoir-faire ou la réputation.

L'idée de l'exploitation et la combinaison des ressources est relativement centrale dans les développements récents, et ce depuis les travaux de Ray, Barney et Muhanna (2004). Selon Weppe, Warnier et Lecocq (2013), au-delà de l'analyse des ressources dites stratégiques permettant aux entreprises de faire la différence, il est important de considérer les ressources ordinaires, qui n'offrent pas la possibilité aux entreprises de faire la différence, et les ressources négatives, qui sont perçues comme potentiellement pénalisantes pour l'organisation. Pour ces auteurs, d'une part la focalisation des études sur les ressources stratégiques ayant les attributs VRIN conduit à se concentrer sur des entreprises ayant réussi (success story) et sur un nombre restreint d'entreprises (Weppe et al., 2012). D'autre part, c'est la prise en compte par l'entreprise des ressources ordinaires et négatives qui engendrerait l'avantage concurrentiel, car elles seraient considérées par un certain nombre d'acteurs comme problématiques, alors que pour d'autres elles présenteraient de la valeur. L'approche proposée par Weppe et ses coauteurs (2013) donne ainsi une importance particulière à la perception du dirigeant concernant la valeur des ressources (Warnier et al., 2015). D'autres travaux ont souligné que la valeur des ressources est déterminée par les connaissances et les croyances des acteurs (Mathews, 2010). Selon Warnier et ses coauteurs (2015), les processus d'évaluation, d'acquisition et d'orchestration des ressources sont liés aux représentations collectives du secteur et de l'acteur (dirigeant ou manager). Le processus de sélection d'une ressource est donc largement influencé par les pratiques des entreprises du secteur. L'intégration de la ressource au business model n'en diffère pas moins d'une organisation à l'autre, ce que souligne Durand (2000, p. 86), « l'entreprise mobilise des actifs et les combine au service de son offre et de ses clients, en faisant appel à des connaissances et des processus organisationnels qui lui sont propres ». De plus, dans le domaine de la RSE, les perceptions et interprétations diverses des acteurs ont été identifiées par de nombreux travaux (Cramer et al., 2004). Cela conforte, s'il en était besoin la nécessité de replacer les acteurs au cœur des processus de valorisation des ressources.

RIMHE - Revue Interdisciplinaire Management, Homme \& Entreprise

n³2 - Eté 2018

-50 - 
Article de recherche : Responsabilité sociétale de l'entreprise : une étude de la nature et de

la valeur perçues des ressources mobilisées dans trois entreprises du secteur de

l'imprimerie - Marie DA FONSECA et Elise BONNEVEUX

Durand (2013) a critiqué l'approche fondée sur les ressources arguant que l'avantage concurrentiel ne provient pas des ressources mais des compétences organisationnelles. Selon l'auteur, c'est la combinaison des ressources et non les ressources elles-mêmes qui peuvent être source d'avantage concurrentiel. Le débat qu'il a introduit nous semble essentiellement montrer l'importance de procéder conjointement à une catégorisation des ressources, et à une étude de leurs perceptions par les acteurs. Dans cette étude, nous nous positionnons donc dans l'approche de Weppe, Warnier et Lecocq (2013) des ressources. Elle permet de sortir de l'analyse rationnelle et instrumentale de l'approche classique, et se présente comme une grille d'intelligibilité permettant d'expliquer pourquoi une même ressource disponible va être employée stratégiquement par une entreprise et, à l'inverse, ne pas être exploitée par d'autres dans un même secteur d'activité.

\subsection{La pertinence de l'approche fondée sur les ressources en matière de RSE}

Le lien entre responsabilité sociétale et les ressources a été précédemment fait dans les travaux portant sur les stratégies environnementales. En 1995, Hart propose un modèle fondé sur les ressources naturelles. Selon cet auteur, les entreprises développent un avantage concurrentiel grâce aux relations qu'elles entretiennent avec leur environnement naturel. Les entreprises adoptent un des trois types de stratégies dites environnementales, à savoir la prévention des pollutions, l'éco-conception et le développement durable. Dans la première, l'entreprise cherche à réduire ses émissions. Il y a une recherche de qualité environnementale qui se traduit par une stratégie de domination par les coûts. Dans la deuxième, l'enjeu est de minimiser les impacts tout au long du cycle de vie du produit. Dans le cadre d'une stratégie d'éco-conception, l'entreprise intègre ses parties prenantes, ce qui lui permet d'anticiper sur la stratégie de ses concurrents. Enfin, la troisième stratégie dite de «développement durable » se traduit par une projection de l'entreprise à long terme qui cherche à minimiser son empreinte environnementale.

A la suite des travaux de Hart (1995), de nombreux auteurs vont développer cette typologie (Russo et Fouts, 1997 ; Sharma et Vredenburg, 1998 ; Christman, 2000 ; Buysse et Verbeke, 2003 ; Bowen et Sharma, 2005 ; Fowler et Hope, 2007). Ces travaux montrent qu'il est alors possible de créer un avantage concurrentiel via le développement de stratégies pro-actives en lien avec le développement durable. Les entreprises peuvent ainsi exploiter des opportunités qui leur permettent de générer des rentes. Les auteurs considèrent les ressources comme importantes dans le développement de stratégies proactives et particulièrement les ressources technologiques (Shrivastava, 1995), les compétences en termes de prévention des pollutions, les compétences managériales, les attitudes et les représentations (Sharma, 2000). Aragon-Correa et Sharma (2003) ont, en outre, mis l'accent sur le fait que les

RIMHE - Revue Interdisciplinaire Management, Homme \& Entreprise

n³2 - Eté 2018 
Article de recherche : Responsabilité sociétale de l'entreprise : une étude de la nature et de

la valeur perçues des ressources mobilisées dans trois entreprises du secteur de

l'imprimerie - Marie DA FONSECA et Elise BONNEVEUX

entreprises devaient porter une attention particulière aux ressources organisationnelles et humaines.

Historiquement, si l'approche par les ressources, issue du champ du management stratégique a été mobilisée, c'est d'abord pour décrire les stratégies environnementales des firmes proactives. L'enjeu est donc à présent d'élargir les études à tous les comportements organisationnels et de s'intéresser aux ressources mobilisées, quel que soit le comportement de l'entreprise face à la responsabilité sociétale. L'approche théorique de Weppe, Warnier et Lecocq (2013) se présente comme une grille d'intelligibilité permettant d'étudier la nature et la valeur perçues par les dirigeants des ressources mobilisées dans le cadre d'une démarche responsable.

\section{Méthodologie de l'étude de cas de trois entreprises}

Cette recherche est de nature exploratoire et compréhensive (Dumez, 2013) et s'inscrit dans une approche interprétativiste et abductive (Yin, 1994 ; Wacheux, 1996). Nous avons mené une étude de cas auprès de trois entreprises familiales, officiant dans le secteur de l'imprimerie.

\subsection{Le choix du secteur d'activité et des trois cas de l'étude}

Le secteur d'activité de l'imprimerie (annexe 1) est particulièrement concerné par la RSE du fait des importants enjeux environnementaux de l'activité. L'apparition d'une réglementation environnementale sur le papier et les encres en 2004 a été l'occasion pour certaines imprimeries de se restructurer en faisant évoluer leurs activités mais également leur offre de produits. De plus, une meilleure prise en compte de la gestion des déchets a permis des économies importantes. Les problématiques traitées ou rencontrées concernent plus spécifiquement les déchets, comme les solvants toxiques ou les effluents aqueux, certains produits n'étant pas acceptés par les réseaux collectifs et/ou éliminés par les stations d'épurations traditionnelles, avec une incitation à utiliser de nouvelles matières moins polluantes et moins dangereuses pour la santé. Un label, Imprim'Vert, a été développé avec pour objectif de permettre aux entreprises le souhaitant de s'engager dans une démarche volontaire et de pouvoir la valoriser. Ces bouleversements et les enjeux de la RSE inhérents à l'imprimerie rendaient particulièrement pertinents le choix de ce secteur.

Nous avons opté pour trois petites et moyennes entreprises familiales (annexe 2), engagées dans une démarche de responsabilité sociétale de l'entreprise. L'entreprise 1 est une petite entreprise familiale de la vallée de l'Arve. Elle est composée de trois actionnaires et de onze salariés. Nous avons interrogé deux des trois actionnaires (dirigeants A et B). L'entreprise 1 a dû se séparer d'une partie de ses salariés entre 2005 et 2010. C'est dans ce contexte économique difficile avec la perte de nombreux clients qu'elle a décidé d'obtenir le label Imprim'Vert. Elle a réussi à être labellisée en

RIMHE - Revue Interdisciplinaire Management, Homme \& Entreprise

n³2 - Eté 2018 
Article de recherche : Responsabilité sociétale de l'entreprise : une étude de la nature et de

la valeur perçues des ressources mobilisées dans trois entreprises du secteur de

l'imprimerie - Marie DA FONSECA et Elise BONNEVEUX

2008, essentiellement par nécessité. C'est sous la pression de son environnement qu'elle s'est résolue à utiliser des encres végétales. Ses déchets toxiques sont également désormais traités par des entreprises spécialisées. Les dirigeants réservent leur temps à la prospection commerciale et au maintien de contrats peu rémunérateurs avec des institutions pour pérenniser l'activité de l'entreprise 1.

L'entreprise 2 a 50 ans d'existence. Située dans la vallée de l'Arve, elle a une antenne basée à Montpellier et compte six salariés. Les dirigeants sont le père (dirigeant $\mathrm{C}$ ) et son fils (dirigeant D). La démarche RSE a été initiée très tôt. Pour ce faire, ils ont réalisé un benchmark auprès de plusieurs groupes institutionnels afin d'acquérir des informations sur le sujet et ainsi s'orienter vers l'achat ou la location de machines plus respectueuses de l'environnement et moins nocives pour les salariés. Les dirigeants se concentrent sur leur cœur de compétence : la création artistique et l'impression de très haute qualité sur grand format.

L'entreprise 3 est basée dans l'Hérault, elle existe depuis 1979 et compte plus de 100 salariés. Elle est dirigée par un frère et une sœur (dirigeante $\mathrm{E}$ ). La démarche de responsabilité sociétale a été initiée il y a un peu plus d'une dizaine d'années, ce qui fait d'elle un précurseur dans la région. La dirigeante est inscrite dans de nombreux réseaux institutionnels et professionnels en lien avec le développement durable. Les actions menées portent autant sur les aspects environnementaux que sociaux. L'entreprise 3 affiche une progression du chiffre d'affaires de $+34 \%$ sur 4 ans. Elle a également vu sa surface s'agrandir de $5000 \mathrm{~m} 2$ en 2015 , en construisant notamment un bâtiment à haute qualité environnementale (HQE).

\subsection{Mode de collecte et d'analyse des données}

Les données ont été collectées entre juin 2013 et octobre 2016 en menant une série d'entretiens semi-directifs et en rassemblant des données secondaires. Nous avons rencontré les dirigeants des trois entreprises à trois reprises, en suivant le principe de la saturation sémantique (Thiétart, 2011). Neuf entretiens au total d'une heure et demie à deux heures ont donc été menés, enregistrés et retranscrits (tableau 1).

Deux guides d'entretien ont été élaborés en appui sur notre revue de littérature (Miles et Huberman, 2003), l'un pour la phase 1 de première prise de contact, l'autre pour les phases 2 et 3 d'approfondissement. Les thèmes retenus sont : la vision de la RSE, les actions développées, les contraintes rencontrées, les solutions trouvées, les démarches réalisées pour mener les actions (annexe 3).

Les données secondaires ont été trouvées sur les sites internet et les réseaux sociaux ou fournies par les entreprises : réponse à des appels d'offres, plaquettes de présentation de l'entreprise ou de la RSE, etc. (annexe 4). 
Article de recherche : Responsabilité sociétale de l'entreprise : une étude de la nature et de la valeur perçues des ressources mobilisées dans trois entreprises du secteur de l'imprimerie - Marie DA FONSECA et Elise BONNEVEUX

Tableau 1 : Dirigeants rencontrés par entreprise et par phase d'entretien

\begin{tabular}{|l|l|l|l|}
\hline & \multicolumn{1}{|c|}{ Phase 1 } & \multicolumn{1}{c|}{ Phase 2 } & \multicolumn{1}{c|}{ Phase 3 } \\
\hline Entreprise 1 & Dirigeant A (frère, 54 ans) & Dirigeant B (frère, 58 ans) & Dirigeant B \\
\hline Entreprise 2 & $\begin{array}{l}\text { Dirigeant C (père, 62 ans) et } \\
\text { Dirigeant D (fils, 38 ans) }\end{array}$ & Dirigeants C et D & Dirigeant D \\
\hline Entreprise 3 & Dirigeante E (sœur, 50 ans) & Dirigeante E & Dirigeante E \\
\hline
\end{tabular}

Pour analyser les données collectées, nous avons eu recours à l'analyse multithématique en mobilisant la méthode de codage définie par Ayache et Dumez (2011). Elle consiste à travailler à partir de thèmes relativement hétérogènes et à établir ensuite des catégories. Nous avons ensuite procédé à une analyse de contenu thématique continue (Miles et Huberman, 2003 ; Dumez, 2013). Pour ce faire, une grille de codage a été élaborée en reprenant les thèmes issus de la revue de la littérature et structurant notre guide d'entretien, mais également avec des thèmes émergeant de l'analyse (tableau 2). Cette méthode permet une analyse plus fine et plus riche du matériau (Paillé et Mucchielli, 2013).

Tableau 2: Catégories d'analyse de contenu

\begin{tabular}{|l|l|}
\hline \multicolumn{1}{|c|}{ Catégorie issue de la littérature } & \multicolumn{1}{|c|}{ Catégorie issue du terrain } \\
\hline $\begin{array}{l}\text { Caractéristiques Entreprise } \\
\text { Perception de la RSE } \\
\text { Valeurs du Dirigeant }\end{array}$ & $\begin{array}{l}\text { Démarches RSE mises en place } \\
\text { Evénement déterminant en lien } \\
\text { avec RSE }\end{array}$ \\
Freins/obstacles rencontrés & \\
Ressources mobilisées & \\
Valeur donnée aux ressources & \\
Resormance & \\
Combinaison des ressources & \\
\hline
\end{tabular}

Nous avons procédé à une analyse par cas puis à une comparaison inter-cas. 
Article de recherche : Responsabilité sociétale de l'entreprise : une étude de la nature et de

la valeur perçues des ressources mobilisées dans trois entreprises du secteur de

l'imprimerie - Marie DA FONSECA et Elise BONNEVEUX

\section{Résultats de l'étude}

L'analyse des résultats met en lumière le rôle de la perception dans l'attribution d'une valeur aux ressources et dans la mise en œuvre de la démarche responsable de l'entreprise.

\subsection{La perception de la responsabilité sociétale de l'entreprise par les dirigeants}

Pour l'entreprise 1, la RSE est subie. L'entreprise s'adapte aux besoins et demandes du marché, en faisant, par exemple, les aménagements techniques nécessaires pour obtenir les labels du type Imprim'Vert: changement des matières premières, renouvellement du parc technique etc. La démarche n'est pas intériorisée, l'action se fait en réaction au fur-et-à-mesure des pressions environnementales de l'écosystème ou institutionnelles, il n'y a pas d'anticipation des évolutions du marché. De ce fait, la direction a assumé de lourds investissements et de profonds changements organisationnels, sans que l'entreprise y soit préparée. Les dirigeants actuels ne perçoivent pas de bénéfices à ces actions, comme le souligne le dirigeant historique.

"J'ai fait tout ce qu'il fallait, j'ai changé les machines, vous savez combien ça m'a coûté? Mais je ne gagne pas plus, oh non, je ne gagne pas plus » (entreprise 1 - dirigeant A).

La RSE n'est pas intégrée à la stratégie d'entreprise comme un pilier de développement, bien que les dirigeants multiplient les actions au sein de différents réseaux d'affaires pour développer leur entreprise. De plus, la RSE est mise en avant dans leur communication sur leur site internet.

L'entreprise 2 s'est tournée vers la RSE pour faire face à la crise que subit l'imprimerie. Le choix stratégique a été de développer de nouvelles offres de produits ou services : photographie, numérique, programmation, boutique en ligne - librairie, conseil en communication, graphisme. Elle est également labellisée Imprim'Vert mais cette information n'est quasiment jamais mentionnée sur les différents supports de communication (site internet, plaquette de présentation de l'entreprise).

"Lorsqu'on nous le demande, pour certains dossiers oui, on le dit, mais sinon non » (entreprise 2 - dirigeant C).

«Je n'avais jamais réfléchi à notre responsabilité sociétale. On fait les actions. Avant on chauffait au poil à bois, aujourd'hui on a la géothermie. Vous savez on fait les choses parce qu'elles nous semblent importantes pas pour se faire valoir » (entreprise 2 - dirigeant D)

Le fils raconte qu'il y a plusieurs années lorsqu'il a commencé dans l'entreprise, son père lui faisait nettoyer les machines.

"L'odeur était désagréable, non seulement l'odeur des encres, mais également des solvants et des produits d'entretien, si ça l'était pour moi, ça l'était pour les salariés » (entreprise 2 dirigeant $\mathrm{D}$ ). 
Article de recherche : Responsabilité sociétale de l'entreprise : une étude de la nature et de

la valeur perçues des ressources mobilisées dans trois entreprises du secteur de

l'imprimerie - Marie DA FONSECA et Elise BONNEVEUX

Les dirigeants se montrent assez critiques face à leurs concurrents qui surfent sur la vague du développement durable.

"On pourrait faire comme X, mais je vais vous dire, aujourd'hui en France il n'existe plus que des encres végétales et des papiers certifiés, donc ça ne tient pas comme argument. Ça n'est pas un choix de leur part, c'est comme ça » (entreprise 2 - dirigeant D).

Cette entreprise fait toutefois partie d'un club d'entreprises dédié au développement durable et propose régulièrement à ses salariés d'assister aux réunions.

«On ne sait pas tout, et on n'est pas les seuls à avoir des idées. Ça n'est pas obligatoire, c'est du volontariat, on leur propose, ils sont libres. En général, on y va à deux ou trois. Cela nous permet ensuite de discuter » (entreprise 2 - dirigeant $\mathrm{D}$ ).

L'entreprise 3 s'est lancée dans la RSE à la suite d'un voyage de plusieurs mois de la dirigeante avant de reprendre avec son frère l'entreprise familiale. Ayant pris conscience des enjeux du développement durable dans le secteur de l'imprimerie, elle ne se voyait pas diriger l'entreprise sans les prendre en compte.,

"L'imprimerie avec les solvants, les encres et les rebus de papier est une entreprise très polluante » (entreprise 3 - dirigeante $\mathrm{E})$.

"Le développement durable est à la source de la compétitivité et de la performance des entreprises » (entreprise 3 - dirigeante E).

«On ne fait pas du développement durable pour un aspect marketing mais parce qu'il nous permet d'être plus compétitif sur notre marché » (entreprise 3 - dirigeante E).

L'entreprise affiche une performance importante, avec un chiffre d'affaires qui a augmenté de plus $34 \%$ entre 2009 et 2014 . Le parc technologique est régulièrement changé pour gagner en productivité et en qualité. La dirigeante importe les bonnes pratiques des autres entreprises issues de secteurs d'activités différents, telles que les journées « vit ma vie », au cours desquelles les salariés changent ponctuellement de service pour être sensibilisés aux contraintes des métiers de leurs collègues.

\subsection{Des différences de perception de la valeur des ressources}

Ces entreprises, appartenant au même secteur d'activités et engagées dans des démarches responsables, ont développé des visions et des logiques d'action différentes. Les dirigeants de l'entreprise 1 ont une perception relativement négative de la responsabilité sociétale, qu'ils considèrent comme coûteuse et contraignante. De plus, elle leur semble chronophage alors que la production est réalisée de plus en plus à flux tendu. S'investir dans des actions de responsabilité sociétale leur demande du temps qui leur fait défaut. De ce fait, les mesures développées n'ont pas de lien entre elles et sont ponctuelles, comme la recherche du label Imprim'Vert ou encore la participation à des actions collectives de promotion du développement durable. Ces actions sont mises en avant dans la communication auprès des prospects et lors des réponses aux appels d'offres. Les dirigeants ont bien perçu les enjeux stratégiques de

RIMHE - Revue Interdisciplinaire Management, Homme \& Entreprise

n³2 - Eté 2018

- 56 - 
Article de recherche : Responsabilité sociétale de l'entreprise : une étude de la nature et de

la valeur perçues des ressources mobilisées dans trois entreprises du secteur de

l'imprimerie - Marie DA FONSECA et Elise BONNEVEUX

la responsabilité sociétale, pour autant l'investissement qu'ils y consacrent reste relativement faible. Ainsi, ils n'ont jamais fait de la démarche RSE un enjeu stratégique de développement de leur entreprise.

"Nous tenons à nos montagnes ! Depuis Janvier 2008, notre imprimerie est labellisée "Imprim 'Vert". Cela signifie que nous faisons retraiter nos déchets toxiques et recycler tous nos consommables. [...] Nos produits usagés sont autant de déchets qui ont un impact important sur l'environnement s'ils ne sont pas traités correctement. Toute notre équipe est consciente que chaque geste compte ». (entreprise 1 - site internet, consulté le 13/06/16)

"On le fait et on le mentionne, parce que sinon, on n'obtient pas les marchés. Vous savez aujourd'hui, les institutions comme les mairies, si vous voulez travailler avec, vous devez montrer patte blanche, mais ils ne vérifient pas et faut voir en interne le gaspillage. C'est de l'hypocrisie tout ça» (entreprise 1 - dirigeant B)

"On nous en demande toujours plus, mais nos conditions de travail à nous, elles ne s'améliorent pas, j'étais plus heureux il y a 10 ans, sans tout ça. On est à flux tendu, le lundi vous regardez le planning de la semaine, il est vide et il se remplit au fur et à mesure. Elle est notre réalité, assurer nos commandes. Le reste c'est du superflu. Vous croyez que les concurrents sur internet, ils se préoccupent du développement durable?»(entreprise 1 dirigeant A)

Pour l'entreprise 2, la responsabilité sociétale n'est pas intégrée au développement stratégique de l'entreprise. Toutefois, elle est considérée comme un outil stratégique potentiel lors de la réponse à un appel d'offres et dans la conquête de nouveaux marchés.

"On ne communique dessus que lorsque l'on nous le demande, dans les appels d'offres par exemple, bien sûr on le mentionne » (entreprise 2 - dirigeant $\mathrm{C}$ ).

"Notre entreprise, soucieuse de l'avenir de nos enfants, s'engage depuis plusieurs années dans des actions qui visent à réduire son empreinte écologique. Les ressources renouvelables de la planète, telles que l'eau, l'air, le bois... s'épuisent rapidement en raison de la croissance démographique et du développement économique. L'utilisation que nous faisons des ressources non renouvelables modifie définitivement notre environnement... [L'entreprise] évolue dans le sens de l'écologie et du développement durable » (entreprise 2 - réponse à un appel d'offres).

La RSE semble reposer sur les valeurs des dirigeants, qui les amènent à développer de nombreuses actions relativement cohérentes et complémentaires entre elles et dans une perspective holistique de la responsabilité sociétale, comme le recours à la géothermie pour chauffer le bâtiment, l'investissement dans des rotatives aux procédés innovants, permettant de réduire les émissions de solvants, la participation des salariés à des conférences sur le développement durable, ou encore l'amélioration des conditions de travail.

«Nous c'est une démarche interne. Pour nous, c'est une philosophie, pour être en accord avec soi-même, c'est chaque jour faire quelque chose » (entreprise 2 - dirigeant $\mathrm{C}$ )

RIMHE - Revue Interdisciplinaire Management, Homme \& Entreprise

n³2 - Eté 2018

- 57 - 
Article de recherche : Responsabilité sociétale de l'entreprise : une étude de la nature et de

la valeur perçues des ressources mobilisées dans trois entreprises du secteur de

l'imprimerie - Marie DA FONSECA et Elise BONNEVEUX

Enfin, l'entreprise 3 a fait de la responsabilité sociétale de l'entreprise un enjeu stratégique. La compétitivité de l'entreprise 3 est corrélée aux actions mises en œuvre.

«[Cela] a propulsé notre croissance, [afin] de devenir leader de l'impression responsable en France » (entreprise 3 - dirigeante E)

Toutes les actions menées au sein de l'entreprise 3 sont pensées dans ce sens, comme par exemple les investissements dans les presses et le mobilier, la formation des commerciaux à l'écoconduite, la construction d'un nouveau bâtiment répondant aux normes Haute Qualité Environnementale (HQE). L'entreprise est également certifiée Imprim'Luxe et Iso 9001 et 14001.

Le rôle des perceptions pour définir la nature et la valeur des ressources ressort de l'analyse inter-cas. Ainsi, concernant le label Imprim'Vert, qui est peu contraignant, peu coûteux et accessible à toutes les entreprises sur le marché, l'entreprise 3 a communiqué très tôt sur son obtention, et bien qu'aujourd'hui de très nombreuses entreprises soient également labellisées, les dirigeants continuent de communiquer en mettant en avant que leur entreprise a été la première de la région à l'avoir obtenu. L'entreprise le considère comme une ressource stratégique. A l'opposé, pour l'entreprise 1, le label est une ressource négative. Les dirigeants n'ont pas intégré la RSE dans leur stratégie et en ont sous-estimé les enjeux. L'obtention du label a nécessité de lourds investissements, puisque l'entreprise a dû changer une partie de son parc machine, obsolète, ce qui a engendré des coûts imprévus. Pour l'entreprise 2, le label a été considéré comme une ressource accessible, c'est-à-dire comme une ressource ordinaire.

Cette même logique se poursuit avec l'obligation d'utiliser des encres végétales ou du papier recyclé, qui est perçue de la même manière comme une ressource négative pour l'entreprise 1, ordinaire pour l'entreprise 2 et stratégique pour l'entreprise 3 . Ainsi, le dirigeant A de l'entreprise 1 perçoit négativement ces ressources puisqu'elles se traduisent par une augmentation des coûts de production et une baisse de prix de facturation, associées à une exigence accrue des clients.

«Dans l'imprimerie ça fait 10 ans que les prix n'ont pas augmenté, rien que ce mois-ci le papier a pris $10 \% »$. (entreprise 1 - dirigeant A)

«On n'a plus le choix, mais par contre le papier certifié ça coûte cher et les clients ils veulent toujours des prix moins chers » (entreprise 1 - dirigeant $\mathrm{B}$ )

Pour les dirigeants de l'entreprise 2, sans qu'il soit nécessaire d'en faire un axe stratégique dans la communication externe à l'égard des clients, il est normal d'utiliser ce type de ressources, d'autant que pour eux :

«Sur le marché, elles sont les seules à être disponibles » (entreprise 2 - dirigeant C).

"Vous allez demander à une imprimerie d'imprimer quelque chose écologique, on a plus le choix depuis 20 ans dans nos produits, c'est un phénomène de mode de communiquer dessus. On n'a pas le choix, on peut prendre plein d'exemples, c'est comme si un pharmacien jetait les

RIMHE - Revue Interdisciplinaire Management, Homme \& Entreprise

n³2 - Eté 2018

-58 - 
Article de recherche : Responsabilité sociétale de l'entreprise : une étude de la nature et de

la valeur perçues des ressources mobilisées dans trois entreprises du secteur de

l'imprimerie - Marie DA FONSECA et Elise BONNEVEUX

médicaments dans une poubelle, il n'a pas le droit. Nous, dans notre secteur on doit utiliser des encres vertes, du papier certifié, il en existe pas d'autre sur le marché ». (entreprise 2 dirigeant $C$ )

Par contre, l'entreprise 3 met sans cesse en avant l'utilisation de ce type de ressources comme axe différenciant vis-à-vis de ses concurrents. Elle publie le nombre de tonnes de papier recyclé et certifié, utilisé chaque année. Misant sur son développement et sa croissance responsable, elle considère la moindre action menée comme un argument marketing et la valorise stratégiquement.

«VRAI OU FAUX! [L'entreprise] fût-elle la première imprimerie du Languedoc-Roussillon à obtenir le label Imprim'Vert? » (entreprise 3 - Facebook - page consultée le 15/07/2016)

"Le saviez-vous? [L'entreprise] n'est pas uniquement engagé pour l'environnement. Entreprise à taille humaine, l'Humain est au cœur de nos principales priorités. Découvrez les différentes actions sociales que nous mettons en place afin d'apporter un lieu de travail sécurisé et confortable à nos employés. » (entreprise 3 - Facebook - page consultée consulté le 15/07/2016)

"Le saviez-vous ? Notre ambition chez [1'entreprise] est de renforcer la valeur ajoutée environnementale de nos offres. Soucieux de l'environnement et afin de protéger notre belle planète, nous mettons en place quotidiennement des actions environnementales. » (entreprise 3 - Facebook - page consultée consulté le 15/07/2016)

Concernant les démarches collectives proposées par les réseaux professionnels, cellesci sont de plus en plus nombreuses pour aider les dirigeants à mettre en place des pratiques de RSE. Le temps à y consacrer et la formation qu'ils proposent sont des ressources perçues, là encore, pour l'entreprise 3 comme étant stratégiques, pour l'entreprise 1 de manière négative. Cette dernière, l'entreprise 1 , est typiquement en déficit de temps, la production à flux tendu et l'impossibilité d'anticiper sur les commandes créent des tensions.

«Le lundi vous regardez, le planning, la fin de semaine est vide, le mardi ça se remplit, le mercredi ça déborde, j'irai bien aux formations proposées par le club, mais je n'ai pas le temps » (entreprise 1 - dirigeant $\mathrm{B})$.

Dans l'entreprise 1, la priorité est donnée aux commandes, et les salariés comme les dirigeants ne bénéficient pas des formations à leur disposition, qui leur permettraient pourtant de développer les relations réticulaires, mais également d'acquérir des bonnes pratiques.

Dans l'entreprise 3, beaucoup de temps est consacré à l'opérationnalisation de la responsabilité sociétale, ainsi qu'à la formation, considérées comme des ressorts stratégiques soutenant la démarche responsable.

«Ça permet de fidéliser les salariés, de les faire adhérer à la démarche, et donc de la diffuser en interne, maintenant rien n'est obligatoire, on propose une formation à l'écoconduite volontaire, un samedi matin et en échange on paie une demi-journée de travail à nos salariés. C'est un cadeau qu'on leur fait » (entreprise 3 - dirigeante E).

RIMHE - Revue Interdisciplinaire Management, Homme \& Entreprise

n³2 - Eté 2018

- 59 - 
Article de recherche : Responsabilité sociétale de l'entreprise : une étude de la nature et de

la valeur perçues des ressources mobilisées dans trois entreprises du secteur de

l'imprimerie - Marie DA FONSECA et Elise BONNEVEUX

L'entreprise 3 mobilise toutes les ressources possibles, accessibles gratuitement mais aussi payantes, et donne du temps aux salariés pour qu'ils puissent s'investir dans la démarche. Les bénéfices lui semblent plus importants que les investissements.

\subsection{Une mobilisation isolée ou combinée des ressources}

Aux différences de perception des mêmes ressources disponibles pour mettre en œuvre une démarche de RSE, considérées comme ordinaires par l'entreprise 2, négatives par l'entreprise 1 et stratégiques par l'entreprise 3, s'ajoutent des différences dans le mode de mobilisation des ressources : combinée pour les entreprises 3 et 2, et isolée pour l'entreprise 1.

En effet, dans l'entreprise 3, plusieurs combinaisons de ressources ont été développées pour faire de la responsabilité sociale un axe stratégique différenciant. Cette combinaison des ressources considérées comme stratégiques a permis à l'entreprise 3 de développer une image d'imprimerie verte.

«Spécialiste de l'impression responsable » (entreprise 3 - site internet)

«Leader de l'imprimerie responsable en France » (entreprise 3 - dirigeante E)

Par contre, bien que l'entreprise 1 dispose de ressources qui pourraient faire l'objet d'une combinaison, servir des arguments commerciaux et participer au développement stratégique de l'entreprise, son business model de départ n'a pas évolué, ce qui engendre une mobilisation isolée des ressources dans le cadre des démarches de responsabilité sociétale.

"Il a fallu changer le parc machine quand on a voulu obtenir le label Imprim 'vert, c'était ça ou on ne travaillait plus avec les institutions. Alors on a investi. Mais vous croyez que je facture plus cher du coup ? Pas du tout, les encres et le papier me coûtent plus mais ils [les clients] sont toujours à discuter sur le prix. Tout est comme ça, on en fait toujours plus et du fait de la concurrence mondiale, on tire sur les prix constamment» (entreprise 1 - dirigeant A) «Je n'ai pas le temps [d'aller aux rencontres et formations proposées par les réseaux professionnels à la suite de l'obtention du label Imprim'vert], ça m'ennuie et ce n'est pas adapté à notre contexte ... je ne sais pas comment le transposer dans mon entreprise » (entreprise 1 - dirigeant B).

La combinaison d'un ensemble de ressources considérées comme stratégiques amène l'entreprise 3 à développer un avantage concurrentiel certain par rapport à ces concurrents, puisque ces ressources deviennent source de nouvelles rentes et ont des coûts d'acquisition inférieurs à la performance qu'elles induisent. Alors que pour l'entreprise 1, les mêmes ressources, non combinées entre elles et parfois non mobilisées, ont une valeur perçue négative. Cela conduit le dirigeant à considérer la responsabilité sociétale comme destructive de valeur pour son entreprise. Enfin, pour l'entreprise 2, les ressources sont combinées selon certaines circonstances, notamment lorsque l'entreprise répond à un appel d'offres et qu'il est explicitement demandé des

RIMHE - Revue Interdisciplinaire Management, Homme \& Entreprise

n³2 - Eté 2018

- 60 - 
Article de recherche : Responsabilité sociétale de l'entreprise : une étude de la nature et de

la valeur perçues des ressources mobilisées dans trois entreprises du secteur de

l'imprimerie - Marie DA FONSECA et Elise BONNEVEUX

éléments en lien avec le développement durable. Pour satisfaire à leur responsabilité sociétale, certaines parties prenantes se montrent de plus en plus exigeantes avec leur fournisseur, a fortiori dans l'imprimerie.

"Aujourd'hui, on utilise tous des encres végétales et des papiers certifiés. On a tous le label Imprim'Vert. Se dire révolutionnaire parce qu'on utilise ce type de produit, c'est un mensonge. On n'a pas le choix et heureusement » (entreprise 2 - dirigeant D)

"On y apprend beaucoup de chose [aux rencontres et formations des réseaux professionnels], les échanges sont riches. C'est de la culture pour nous, rien de plus » (entreprise 2 - dirigeant C).

«Bien sûr qu'on communique quand c'est nécessaire. On a un parc machine innovant qui permet de réduire les chutes de papiers, d'imprimer sur de nouveaux supports. On propose des prestations de qualité. On est membre de plusieurs réseaux, ce qui nous conduit à mener des actions collectives pour sauvegarder la montagne. Pour gagner des clients, on sait le mettre en avant. Mais pas dans notre communication de tous les jours » (entreprise 2 - dirigeant C).

\section{Discussion}

Nos résultats confortent l'approche théorique des ressources de Weppe, Warnier et Lecocq (2013), considérant qu'il existe plusieurs perceptions d'une même ressource selon le contexte organisationnel dans lequel elle est mobilisée. Notre analyse montre, dans le cadre d'une démarche de responsabilité sociétale, que la perception de la nature et de la valeur d'une ressource varie en fonction des dirigeants. Les répercussions pour l'entreprise en ressortent comme importantes. En effet, cette perception des dirigeants va déterminer le business model de l'entreprise et favoriser ou non son développement et sa capacité à s'appuyer sur les ressources externes mises à sa disposition. Le constat que l'entreprise 3 combine les ressources disponibles pour mettre en œuvre une démarche de RSE qu'elle perçoit par ailleurs comme stratégique, tandis que l'entreprise 1 sollicite ces ressources de façon isolée alors qu'elle les perçoit négativement, nous conduit à formuler deux propositions de recherche: 1) les ressources perçues comme stratégiques auront tendance à être combinées ; 2) les ressources perçues comme négatives auront tendance à être mobilisées de façon isolée.

Un processus ressort également de notre étude que nous nous proposons de modéliser (figure 1), celui de la construction de la valeur perçue des ressources dans le cadre d'une démarche de responsabilité sociétale de l'entreprise. En effet, le niveau de performance de l'entreprise peut être considéré à la fois comme une résultante et un déterminant de la valeur perçue des ressources par les dirigeants. Il va dépendre de la mobilisation des ressources, lui-même fonction de la perception des dirigeants, qu'il va, en même temps, venir renforcer.

RIMHE - Revue Interdisciplinaire Management, Homme \& Entreprise

n³2 - Eté 2018

-61 - 
Article de recherche : Responsabilité sociétale de l'entreprise : une étude de la nature et de

la valeur perçues des ressources mobilisées dans trois entreprises du secteur de

l'imprimerie - Marie DA FONSECA et Elise BONNEVEUX

Figure 1 : Construction de la valeur perçue d'une ressource dans une démarche RSE

\begin{tabular}{|c|c|c|c|}
\hline Secteur & Dirigeant & Organisation & \\
\hline $\begin{array}{l}\text { Perception inter } \\
\text { subjective de la RSE } \\
\text { Perception inter } \\
\text { subjective de la } \\
\text { ressource }\end{array}$ & $\begin{array}{l}\text { Perception } \\
\text { subjective de la } \\
\text { RSE } \\
\text { Perception } \\
\text { subjective de la } \\
\text { ressource }\end{array}$ & 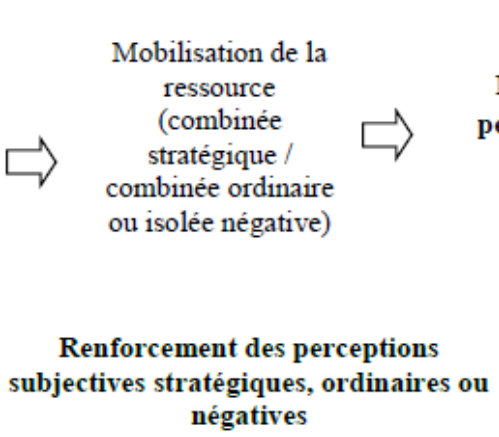 & $\begin{array}{c}\text { Niveau de } \\
\text { performance }\end{array}$ \\
\hline
\end{tabular}

Ainsi, l'entreprise 2 a choisi de diversifier son activité, tout en développant des actions ponctuelles sans que la RSE ne soit intégrée dans la stratégie de l'entreprise. Ses critères de performance sont avant tout économiques, bien que l'harmonie dans l'entreprise et le bien-être des salariés soient une des préoccupations des dirigeants. Ceux-ci positionnent comme ordinaires les ressources mises à disposition pour développer une démarche de responsabilité sociétale. Ils ne se réfèrent qu'à quelques indicateurs sans vision globale, comme la quantité de déchets, les économies d'énergies, la satisfaction client ou encore la qualité. Pour l'entreprise 2, la RSE est une philosophie, intrinsèque à l'entreprise et reliée à leurs valeurs, elle ne fait l'objet d'aucun plan stratégique. Par contre, elle permet, de fait, de motiver les salariés ou de réaliser des économies. En ce sens, elle est ponctuellement considérée comme un outil stratégique, sur lequel s'appuyer pour infléchir le jugement et la prise de décision de prospects ou remporter un appel d'offres. Si l'entreprise a proposé de nouveaux produits ou services répondant aux exigences d'un développement durable concernant les solvants toxiques, c'est dans une démarche d'innovation et non de RSE. Pour les dirigeants de l'entreprise 2, prôner une stratégie de responsabilité sociétale serait instrumentaliser leurs propres valeurs à des fins économiques. Ainsi, c'est la diversification des activités, associée à une production haut de gamme et de qualité, qui est un axe de développement stratégique afin d'assurer l'accroissement de la performance, et non la RSE.

En contraste, l'entreprise 3 mise tout son argumentaire sur la démarche responsable. Ses critères de performance sont tant économiques, écologiques que sociaux. Les dirigeants s'appuient sur une multitude de critères: diversité, égalité, handicap, 
Article de recherche : Responsabilité sociétale de l'entreprise : une étude de la nature et de

la valeur perçues des ressources mobilisées dans trois entreprises du secteur de

l'imprimerie - Marie DA FONSECA et Elise BONNEVEUX

croissance, investissements, énergies, déchets, bâtiments, conditions de travail, etc. La responsabilité sociétale est une partie intégrante du plan de développement stratégique de l'entreprise. En positionnant l'entreprise comme leader de l'imprimerie verte, les dirigeants ont fait de la responsabilité sociétale un des piliers du business model de l'entreprise 3.

Les dirigeants de l'entreprise 1 considèrent la performance principalement comme financière avec une vision à court-terme d'une activité à flux tendu. Leur vision du marché les conduit à aligner leurs prix sur ceux de la concurrence en les tirant vers le bas, alors que les entreprises 3 et 2, en misant sur la qualité, associée respectivement, pour l'une, à une démarche de responsabilité sociétale, pour l'autre, à l'innovation, ont pu augmenter leurs tarifs et ainsi absorber les investissements réalisés en réponse aux exigences du développement durable. La RSE est perçue comme une contrainte par les dirigeants de l'entreprise 1, mais sous la pression de leur environnement et des clients, ses dirigeants se sont résolus à accepter de jouer le jeu sans développer une réelle stratégie de responsabilité sociétale. Ils perçoivent négativement et ne combinent pas les ressources à leur disposition, considérant qu'elles coûtent plus que ce qu'elles rapportent.

\section{Conclusion}

Nos résultats ont permis de clarifier la nature des ressources mobilisées dans le cadre d'une démarche responsable. Il est ressorti que les trois entreprises de l'étude, relevant du même secteur d'activité, avait à disposition les mêmes ressources externes mais que leurs divergences de perception de la valeur de ces ressources les conduisaient à les mobiliser différemment. Nous montrons ainsi qu'une même ressource peut être perçue comme stratégique, ordinaire ou négative par les dirigeants d'entreprise. Ce sont donc les perceptions des ressources par les dirigeants et non leur nature et leur valeur intrinsèques qui confèrent ou non un avantage concurrentiel aux entreprises. Ce résultat débouchant sur la proposition d'une modélisation du processus de construction de la valeur d'une ressource représente notre principale contribution. Il conforte et approfondit l'approche théorique des ressources de Weppe, et (2013), tout en se présentant comme une grille d'intelligibilité des divergences constatées dans la mise en œuvre des démarches responsables par des entreprises relevant d'un même secteur d'activité.

Notre étude présente plusieurs limites associées aux cas étudiés et à la nature exploratoire de notre recherche. Ainsi, le contexte des entreprises sélectionnées peut être considéré comme favorable à la mise en évidence du rôle des perceptions des dirigeants. Les ressources disponibles ne sont certainement pas accessibles de la même façon dans d'autres pays ou pour d'autres secteurs d'activité. Par ailleurs, si les trois entreprises concernées étaient des petites entreprises familiales afin d'en permettre la

RIMHE - Revue Interdisciplinaire Management, Homme \& Entreprise

n³2 - Eté 2018

-63 - 
Article de recherche : Responsabilité sociétale de l'entreprise : une étude de la nature et de

la valeur perçues des ressources mobilisées dans trois entreprises du secteur de

l'imprimerie - Marie DA FONSECA et Elise BONNEVEUX

comparaison, nous n'avons pas pu analyser l'impact de cette caractéristique sur nos résultats. Nos résultats débouchent ainsi sur des pistes de recherche auprès d'une population plus large d'entreprises, ou encore en collectant les perceptions de l'ensemble des parties prenantes de l'écosystème constitué par la mise en œuvre d'une démarche de RSE.

Enfin, cette recherche amène à considérer que les réticences de la part de certains dirigeants d'entreprise à la mise en place de démarche de responsabilité sociétale pourraient être levées, si les discours institutionnels ne portaient plus essentiellement sur les success story, mais sur la nécessaire combinaison des ressources et le développement des compétences pour parvenir à construire une responsabilité sociétale stratégique.

\section{Références}

Acquier A., Gond J.-P. (2005), Aux sources de la responsabilité sociale de l'entreprise relecture et analyse d'un ouvrage séminal : social responsibilities of the businessman d'Howard Bowen, Finance Contrôle Stratégie, vol.10, n², p.5-35.

Acquier A. (2007), RSE et outils de gestion : Chaîne d'outils et infrastructure instrumentale des marchés de l'évaluation extra-financière, Revue de l'Organisation Responsable, vol.2, $\mathrm{n}^{\circ}$ 2, p.5-15.

Acquier A., Dalmasso C. (2009), Vers une approche dynamique de la théorie des ressources, le cas de l'internationalisation des ressources d'ingénierie chez un constructeur automobile, Actes de la Conférence Internationale de Management Stratégique de l'AIMS, Grenoble.

Acquier A. (2010), CSR in search of a management model, in Smith N.C., Bhattacharya C.B., Vogel D., Levine D.I., Global Challenges in Responsible Business, Cambridge, Cambridge University Press, p.107-132.

Amit R.H., Schoemaker P.H., (1993), Strategic assets and organizational rent, Strategic Management Journal, vol.14, $\mathrm{n}^{\circ} 1$, p.33-46.

Aragon-Correa J., Sharma S. (2003), A contingent resource-based view of proactive Activities, and Routines in Disequilibrium World, Organization Studies, vol. 31, n 1 , p.219-244.

Auberger M.N., Quairel F. (2004), Des PME socialement responsables ?, Economie \& Humanisme, $\mathrm{n}^{\circ} 370$, p. 16-19.

Ayache M., Dumez H. (2011), Réflexions en forme de réponses : à propos du codage dans la recherche qualitative, Le Libellio d'AEGIS, vol. 7, n³, p. 29-34.

Barney J. (1991), Firm resources and sustained competitive advantage, Journal of Management, vol.17, $\mathrm{n}^{\circ} 1, \mathrm{p} .99-120$.

RIMHE - Revue Interdisciplinaire Management, Homme \& Entreprise

n³2 - Eté 2018

- 64 - 
Article de recherche : Responsabilité sociétale de l'entreprise : une étude de la nature et de

la valeur perçues des ressources mobilisées dans trois entreprises du secteur de

l'imprimerie - Marie DA FONSECA et Elise BONNEVEUX

Barney J.B. (2001), The resource-based view of the firm: ten years after 1991, Journal of Management, $\mathrm{n}^{\circ}$ 6, p.643-650.

Bowen H.R. (1953), Social responsibilities of the businessman, New York, Harper et Row.

Bowen F.E., Sharma S. (2005), Resourcing corporate environmental strategy: behavioural and resource-based perspectives, Academy of Management, vol.2005, $\mathrm{n}^{\circ} 1$ - https://doi.org/10.5465/ambpp.2005.18783302

Buysse K., Verbeke A. (2003), Proactive Environmental Strategies: A Stakeholder Management Perspective, Strategic Management Journal, vol.24, p.453-470.

Capron M., Quairel-Lanoizelée F. (2004), Mythes et réalités de l'entreprise responsable, Paris, La Découverte.

Clark C.E. (2000), Differences between public relations and corporate social responsibility: an analysis, Public Relation Review, vol.26, n 3, p.363-380.

Conner K.R., Prahalad C.K. (1996), A resource-based theory of the firm: knowledge versus opportunism, Organization Science, vol. 7, $\mathrm{n}^{\circ}$ 5, p. 477-501.

Cramer J., Jonker J., Van der Hejiden A. (2004), Making Sense of Corporate Social responsibility, Journal of Business Ethics, vol.55, n ${ }^{\circ}$, p.215-222.

Dumez H. (2013), Méthodologie de la recherche qualitative, les 10 questions clés de la démarche compréhensive, Paris, Vuibert.

Durand T. (2000), L'alchimie de la compétence, Revue Française de Gestion, $\mathrm{n}^{\circ}$ 127, p.84-102.

Durand T. (2013), Une critique de l'article 'Ressources stratégiques, ressources ordinaires et ressources négatives', Revue Française de Gestion, n 234, p.64-73.

Fowler S.J., Hope C. (2007), Incorporating Sustainable Business Practices into Company Strategy, Business Strategy and Environment, vol.16, ${ }^{\circ} 1, \mathrm{p} .26-38$.

Frederick W.C. (1978), From CSR 1 to CSR 2: the maturing of business and society thought, Business and Society, vol.33, n², p.150-164.

Gendron C. (2000), Le questionnement éthique et social de l'entreprise dans la littérature managériale, Cahier $d u$ CRISES, $\mathrm{n}^{\circ} 004$.

Gond J.-P., Matten D. (2007), Rethinking the business-society interface: Beyond the functionalist trap, ICCSR Research paper series, $\mathrm{n}^{\circ} 47$.

Hart S.L. (1995), A natural-resource-based view of the firm, Academy of Management Review, vol.20, n 4, p.986-1014.

Havard C., Ingham M. (2017), Le rôle des managers intermédiaires dans le déploiement d'un programme RSE: le cas du Groupe La Poste, Revue de l'Organisation Responsable, vol. 12, n², p. 5-24.

Helfat C.E., Peteraf M.A. (2003), The dynamic resource-based view: capability lifecycles, Strategic Management Journal, vol.24, n ${ }^{\circ}$, p.997-1010.

RIMHE - Revue Interdisciplinaire Management, Homme \& Entreprise

n³2 - Eté 2018

-65 - 
Article de recherche : Responsabilité sociétale de l'entreprise : une étude de la nature et de la valeur perçues des ressources mobilisées dans trois entreprises du secteur de l'imprimerie - Marie DA FONSECA et Elise BONNEVEUX

Hine J., Preuss L. (2009), Society is Out There, Organization is in Here: On the Perceptions of Corporate Social Responsibility Held by Different Managerial Group, Journal of Business Ethics, vol.88, n² 2 , p.381-393

Johnson R.A., Greening D.W. (1999), The Effects of Corporate Governance and Institutional Ownership Types on Corporate Social Performance, Academy of Management Journal, vol.42, n5, p.564-576.

King A., Lenox M. (2000), Industry Self-Regulation Without Sanctions: The Chemical Industry's Responsible Care Program, Academy of Management Journal, vol.43, n 4 , p.608-716.

Maon F., Lindgreen A., Swaen V. (2008), Thinking of the organization as a system: the role of managerial perceptions in developing a corporate social responsibility strategic agenda, Systems Research and Behavioral Science, vol.25, n³, p.413-426.

Marais M., Reynaud E. (2008), Comparaison entre les entreprises françaises publiques et privées face aux exigences du développement durable, Management International, vol.12, $\mathrm{n}^{\circ} 4, \mathrm{p} .45-69$.

Mathews J.A. (2010), Lachmannian Insights into Strategic Entrepreneurship: Resources, activities and routines in a disequilibrium world, Organization Studies, vol.31, n², p.219-244.

Mathieu A., Soparnot R. (2009), Les dimensions ontologiques, stratégiques et organisationnelles de l'appropriation du concept de développement durable en entreprise, Management \& Avenir, vol. 3, n 23, p. 199-218.

Métais E. (2004), Stratégie et ressources de l'entreprise. Théorie et pratique, Paris, Economica.

Miles M.B., Huberman A.M. (2003), Analyse des données qualitatives, Bruxelles, De Boeck Supérieur.

Miller D., Shamsie J. (1996), The resource-based view of the firm in two environments: the Hollywood film studios from 1936 to 1965, Academy of Management Journal, vol.39, n`3, p.519-536.

Nielsen A.E., Thomsen C. (2007), Reporting CSR: what and how to say it?, Corporate Communications, vol.12, $\mathrm{n}^{\circ} 1, \mathrm{p} .25-40$.

Paillé P., Mucchielli A. (2013), L'analyse qualitative en sciences humaines et sociales, Paris, Armand Colin.

Paradas A. (2007), Le dirigeant comme levier de la RSE en TPE. Approche exploratoire basée sur l'utilisation de récits et d'une cartographie cognitive, Revue Internationale PME, vol.20, ${ }^{\circ}$ 3-4, p.43-67.

Pedersen E. (2010), Modelling CSR: How Managers Understand the Responsibilities of Business Towards Society, Journal of Business Ethics, vol. $91 \mathrm{n}^{\circ}$ 2, p.155-166.

Peteraf M., Barney J.B. (2003), Unraveling the resource-based tangle, Managerial and Decision Economics, vol.24, n 4, p.309-323.

RIMHE - Revue Interdisciplinaire Management, Homme \& Entreprise

n³2 - Eté 2018

- 66 - 
Article de recherche : Responsabilité sociétale de l'entreprise : une étude de la nature et de

la valeur perçues des ressources mobilisées dans trois entreprises du secteur de

l'imprimerie - Marie DA FONSECA et Elise BONNEVEUX

Porter M.E. (1991), Towards a Dynamic Theory for Strategy, Strategic Management Journal, vol.12, n' ${ }^{\circ} 2$, p.95-117.

Priem R.L., Butler J.E. (2001), Is the resource-based 'view' a useful perspective for strategic management research?, Academy of Management Review, vol.26, ${ }^{\circ} 1$, p.2240.

Ray G. Barney J.B., Muhanna W. (2004), Capabilities, business processes, and competitive advantage: choosing the dependent variable in empirical tests of the Resource-Based View, Strategic Management Journal, vol. 25, n¹, p. 23-37.

Russo M.V., Fouts P.A. (1997), A Resource-Based Perspective on Corporate Environmental Performance and Profitability, Academy of Management Journal, vol.40, ${ }^{\circ} 3$, p.534-559.

Saulquin J.-Y., Schier G. (2007), Responsabilité sociétale des entreprise et performance : complémentarité ou substitualité ?, Revue des Sciences de Gestion, $\mathrm{n}^{\circ} 233, \mathrm{p} .57-65$.

Sharma S. (2000), Managerial interpretations and organizational context as predictors of corporate choice of environmental strategy, Academy of Management Journal, vol.43, n ${ }^{\circ} 4$, p.681-697.

Sharma S., Vredenburg H. (1998), Proactive Corporate Environmental Strategy and the Development of Competitively Organizational Capabilities, Strategic Management Journal, vol. $19, \mathrm{n}^{\circ} 8$, p.728-753.

Shrivastava P. (1995), The role of corporations in achieving ecological sustainability, Academy of Management Review, vol.20, ${ }^{\circ} 4$, p.936-960.

Spence L., Rutherfoord J.R., Blackburn R.A. (1998), Small Business and Environmental Issues in the UK and the Netherlands: A Literature Review and Research Agenda, Kingston, Kingston University.

Thiétart R.A. (2011), Méthodes de recherche en management, Paris, Dunod.

Wacheux F. (1996), Méthodes Qualitatives et Recherche en Gestion, Paris, Economica.

Warnier V., Lecocq X., Demil B. (2015), Théories, cadres d'analyse (frameworks) et outils en gestion, Le libellio d'Aegis, vol. 11, $\mathrm{n}^{\circ}$ 4, p. 63-71.

Weppe, X., Warnier, V., Lecocq, X., Fréry, F. (2012), Quand les postulats d'une théorie induisent de mauvaises pratiques : La «théorie des ressources » selon J.B. Barney, Revue Française de Gestion, ${ }^{\circ}$ 228-229, p.253-268.

Weppe X., Warnier V., Lecocq X. (2013), Ressources stratégiques, ressources ordinaires et ressources négatives. Pour une reconnaissance de l'ensemble du spectre des ressources, Revue Française de Gestion, n 234 , p.43-63.

Wernerfelt B. (1984), A Resource-Based View of the firm, Strategic Management Journal, vol. 5, n², p.171-180.

RIMHE - Revue Interdisciplinaire Management, Homme \& Entreprise

n³2 - Eté 2018

- 67 - 
Article de recherche : Responsabilité sociétale de l'entreprise : une étude de la nature et de

la valeur perçues des ressources mobilisées dans trois entreprises du secteur de

l'imprimerie - Marie DA FONSECA et Elise BONNEVEUX

Wood D.J. (1991), Corporate social performance revisited, Academy of Management Review, vol.16, n4, p.691-718.

Yin R. (1994), Case study research: Design and methods, Thousand Oaks, Sage.

\section{Annexe 1 : Le secteur de l'imprimerie}

Le secteur de l'imprimerie est un secteur en profonde mutation avec l'arrivée du numérique qui concurrence désormais le secteur de l'édition et celui de l'impression. De plus, il se situe au cœur des enjeux du développement durable avec la quête permanente de la réduction des déchets et spécifiquement du papier. Il a connu une sévère crise au début des années 2000 qui l'a contraint à se restructurer en faisant évoluer ses activités. Selon l'Institut d'Economie Publique (2015), la production globale des documents imprimés est en recul de $4 \%$ sur l'année 2015, comparativement à 2014. La facturation, quant à elle, subit une baisse de $0,8 \%$, tandis que le prix des matières premières (papier, encres, etc.) augmente, ce qui fragilise encore davantage ce secteur, dont les marges ne font que baisser. Sur une échelle temporelle plus large, entre 2006 et 2015, les prix de vente ont perdu plus de 10\% (INSEE, 2017). En France, le secteur est majoritairement occupé par des TPE/PME familiales qui doivent affronter des grands groupes internationaux. Bien que la concurrence soit mondiale, les imprimeurs interviennent principalement sur des marchés locaux. En effet, si les importations de produits imprimés ne cessent d'augmenter, les exportations diminuent grandement. Selon l'INSEE (2017), actuellement moins de 3.500 entreprises se répartissent le marché de l'imprimerie en France, soit $34 \%$ de moins qu'en 2000. L'intensité concurrentielle et les barrières à l'entrée conduisent les entreprises à une concurrence effrénée pour conquérir et fidéliser les clients. Pour résister, les imprimeries sont amenées à renouveler sans cesse leur parc technologique en faisant l'acquisition de machines à impression jet d'encre numérique qui permettent une meilleure personnalisation des impressions et la diminution des déchets et de l'utilisation de solvants. Pour rester concurrentielles, les entreprises développent également des services (graphisme, communication) et inventent de nouveaux produits ou services (impression à la demande, webto-print, nouveaux supports, personnalisation...). Si la disparition de nombreux concurrents pourrait être une aubaine pour les entreprises restantes, elles doivent faire face à une demande de plus en plus exigeante, la concurrence que se mènent les derniers acteurs sur le marché conduit à une baisse des prix, ce qui incite les clients à demander de plus en plus d'impressions de qualité et plus rapidement. Les entreprises de l'imprimerie ont connu une période charnière, au cours de laquelle seules les plus solides, ou en capacité d'évoluer, ont résisté. Ainsi, le secteur a dû se réinventer, via la création de nouveaux services, comme par exemple la programmation ou le graphisme. 
Article de recherche : Responsabilité sociétale de l'entreprise : une étude de la nature et de la valeur perçues des ressources mobilisées dans trois entreprises du secteur de

l'imprimerie - Marie DA FONSECA et Elise BONNEVEUX

Annexe 2 : Les trois entreprises de l'étude

\begin{tabular}{|l|c|c|c|c|}
\hline & Année de création & $\begin{array}{c}\text { Nombre de } \\
\text { salariés }\end{array}$ & Direction & CA (2017) \\
\hline Entreprise 1 & 1972 & 11 & $\begin{array}{c}3 \text { associés (2 frères et un } \\
\text { associé après un rachat) }\end{array}$ & 2,5 millions \\
\hline Entreprise 2 & 1968 & 6 & 2 associés (Père et Fils) & 1,3 millions \\
\hline Entreprise 3 & 1979 & 113 & 2 associés (Frère et Sœur) & 18,6 millions \\
\hline
\end{tabular}

\section{Annexe 3 : Guide d'entretien de la phase 1 de découverte}

\begin{tabular}{|l|l|}
\hline $\begin{array}{l}\text { Présentation de } \\
\text { l'entreprise et du } \\
\text { dirigeant }\end{array}$ & $\begin{array}{l}\text { Pouvez-vous présenter l'entreprise, son activité, son histoire ? } \\
\text { Pouvez-vous vous présenter (votre parcours)? } \\
\text { Depuis quand êtes-vous sensibilisé aux concepts de développement } \\
\text { durable et de RSE ? }\end{array}$ \\
\hline $\begin{array}{l}\text { Positionnement de } \\
\text { l'entreprise par } \\
\text { rapport à son } \\
\text { RSE }\end{array}$ & $\begin{array}{l}\text { - Le développement durable a-t-il une importance dans votre secteur? } \\
\text { Dans quelle mesure ? } \\
\text { - Dans votre secteur, quels seraient les risques que supporterait une } \\
\text { entreprise qui ne serait pas engagée sur le plan de la RSE ? } \\
\text { - Quels seraient les bénéfices qu'obtiendrait une entreprise qui serait } \\
\text { engagée sur le plan de la RSE ? } \\
\text { - Estimez-vous être plus engagé que vos concurrents ? Pour quelles } \\
\text { raisons? }\end{array}$ \\
\hline $\begin{array}{l}\text { Vision et } \\
\text { représentations de la } \\
\text { RSE }\end{array}$ & $\begin{array}{l}\text { - Comment définissez-vous la RSE dans votre entreprise ? } \\
\text { - Quelles sont les raisons qui ont motivé la mise en place de pratiques } \\
\text { liées à la RSE ? } \\
\text { - Quelles sont les principales valeurs sur lesquelles vous communiquez } \\
\text { ? }\end{array}$ \\
\hline $\begin{array}{l}\text { Champ d'application } \\
\text { de la RSE }\end{array}$ & $\begin{array}{l}\text { - Comment avez-vous procédé pour mettre en place des démarches de } \\
\text { RSE ? } \\
\text { - Avez-vous éventuellement rencontré des difficultés au cours de cette }\end{array}$ \\
\hline
\end{tabular}

RIMHE - Revue Interdisciplinaire Management, Homme \& Entreprise

n³2 - Eté 2018

- 69 - 
Article de recherche : Responsabilité sociétale de l'entreprise : une étude de la nature et de la valeur perçues des ressources mobilisées dans trois entreprises du secteur de

l'imprimerie - Marie DA FONSECA et Elise BONNEVEUX

Annexe 4 : Source des données secondaires par entreprise

\begin{tabular}{|c|c|c|}
\hline Entreprise 1 & Entreprise 2 & Entreprise 3 \\
\hline $\begin{array}{l}\text { Communication sur la } \\
\text { démarche RSE sur le site } \\
\text { internet } \\
\text { Plaquette de présentation de } \\
\text { l'entreprise et de la RSE }\end{array}$ & $\begin{array}{l}\text { Exemple de réponse à appel } \\
\text { d'offres } \\
\text { Communication sur la } \\
\text { démarche RSE sur le site } \\
\text { internet) }\end{array}$ & $\begin{array}{l}\text { Rapport d'activité RSE } \\
\text { Exemple de réponse à appel } \\
\text { d'offre } \\
\text { Communication institutionnelle } \\
\text { sur la démarche RSE sur le site } \\
\text { internet et les réseaux sociaux } \\
\text { Plaquette de présentation de } \\
\text { l'entreprise et de la RSE }\end{array}$ \\
\hline
\end{tabular}

